\title{
Un bon exercice de lecture du Cours de linguistique générale
}

\author{
Claire A. Forel \\ Université de Genève, Suisse \\ claire.forel@unige.ch
}

Résumé: Cet article va rendre compte d'aspects de la transmission de la pensée saussurienne qui ont surgi lors de la préparation et du déroulement (en cours actuellement) d'un séminaire intitulé Reading Saussure and Chomsky. Comme Chomsky n'avait pu lire que le seul CLG, nous prendrons cet ouvrage comme point de repère que nous confronterons aux notes des étudiants, à celles de Saussure ainsi qu'à la Collation Sechehaye (Sofia 2015) récemment parue. Nous prendrons trois passages : les premières lignes du CLG; le fameux circuit de la parole (tous deux dans l' «Introduction» du CLG), et la célèbre comparaison de la langue avec le jeu d'échecs (CLG: 125 et 153) qui avait retenu l'attention de Chomsky. Nous verrons des exemples d'adjonction ou de suppression par rapport aux notes des étudiants, des reformulations qui mettent une meilleure lecture de la pensée saussurienne, ainsi que de+s modifications aux schémas notés par les auditeurs apportés par Bally et Sechehaye. Nous verrons aussi comment Chomsky a choisi de retenir ce qui l'intéressait dans l'œuvre de Saussure, quitte à laisser de côté des aspects qui auraient dû l'interpeler

Mots clés: Saussure \& Chomsky, CLG, langue-parole, compétence-performance

\section{A good reading exercise of the General Linguistics Course}

\begin{abstract}
This article will account for aspects of the transmission of Saussurean thought that arose during the preparation and running (currently under way) of a seminar entitled Reading Saussure and Chomsky. As Chomsky was able to read only the CLG, we will take this book as a reference point that we will compare with the students' notes, those of Saussure and the recently published Sechehaye Collation (Sofia 2015). We will take three passages: the first lines of the CLG; the famous speech circuit (both in the "Introduction" of the CLG), and the famous comparison of the language with the chess game (CLG: 125 and 153) that had attracted the attention of Chomsky. We shall see examples of addition or deletion in relation to the students' notes, reformulations which give a better reading of Saussurean thought, as well as modifications to the schemes noted by the auditors brought by Bally and Sechehaye. We shall also see how Chomsky chose to retain his interest in Saussure's work, leaving aside aspects that should have called him
\end{abstract}

Keywords: Saussure \& Chomsky, CLG, langue-parole, competence-performance 
Comment décliner Saussure lorsque l'on est rattachée à la linguistique anglaise, dans une faculté où, mis à part une brève mention dans un cours de première année, le linguiste genevois n'est plus au programme? Il s'agit à la fois de présenter une approche nouvelle pour les étudiants et de la rattacher à différentes problématiques, comme celle de sa réception $(\mathrm{La}$ réception de Saussure en Amérique du Nord) ou des problèmes posés par sa traduction en anglais (Les traductions anglaises du Cours de linguistique générale). La méthode retenue a toujours été une lecture aussi près du texte que possible, même s'il ne s'agissait que de passages choisis compte-tenu des contraintes de l'enseignement. Néanmoins l'exercice s'est révélé fructueux. Les problèmes de traduction, par exemple, ont permis à une étudiante de remarquer que des deux équivalences offertes en anglais pour ce que Saussure appelle les deux faces du signe, la version de $\mathrm{W}$. Baskin, qui recourt au mot side, est plus convaincante que la retraduction de $\mathrm{R}$. Harris qui choisit l'expression two complementary facets, notamment parce que facet implique plus que deux côtés, ce qui n'est pas (forcément) le cas de side, comme dans the two sides of a coin ou of a piece of paper ${ }^{1}$. Ce même séminaire avait aussi donné l'occasion de remarquer une différence dans l'endroit où se situait le lien connectant la langue et la masse parlante d'une part au facteur temps d'autre part. En effet, il était apparu que la formule retenue dans le $C L G^{2}$, ce lien étant situé à la jonction entre la masse parlante et la langue, était le fait de Bally et Sechehaye, puisque les notes des étudiants le plaçaient à la hauteur de la seule masse parlante, alors que dans les notes de Saussure on le trouvait au niveau de la langue; ceci avait permis à un doctorant, Th. Robert, de rédiger un article sur le facteur temps et ses interprétations graphiques ${ }^{3}$.

Cet article va rendre compte d'aspects de la transmission de la pensée saussurienne qui ont surgi lors de la préparation et du déroulement (en cours actuellement) d'un séminaire intitulé Reading Saussure and Chomsky ${ }^{4}$. Comme Chomsky n'avait pu lire que le seul $C L G^{5}$, nous prendrons cet ouvrage comme point de repère que nous confronterons aux notes des étudiants, à celles de Saussure ${ }^{6}$ ainsi qu'à la Collation Sechehaye (Sofia 2015) récemment parue. Nous prendrons trois passages: les premières lignes du CLG; le fameux circuit de la parole (tous deux dans $l^{\prime}$ «Introduction» du CLG), et la célèbre comparaison de la langue avec le jeu d'échec (CLG: 125 et 153) qui avait retenu l'attention de Chomsky. Nous verrons des exemples d'adjonction ou de suppression par rapport aux notes des étudiants, des reformulations qui mettent une meilleure lecture de la pensée saussurienne, ainsi que des modifications aux schémas notés par les auditeurs apportés par

1 La comparaison avec la feuille de papier est de Saussure. <pour l'article issu des réflexions amenées par ce séminaire, voir Forel (2012).

2 Dorénavant nous citerons ce livre sous cet acronyme et non pas sous le nom de Saussure. Nous nous référons à Saussure (1972). Nous utiliserons aussi l'édition critique de R. Engler (Saussure 1968), abré-gée CLG/E.

3 Du reste, la parution toute récente de Sofia (2015) La «Collation Sechehaye» du 'Cours de linguistique générale' de Ferdinand de Saussure montre que la première intention des éditeurs était de respecter le schéma tel que Saussure l'avait présenté.

4 Donné au semestre d'automne 2016 à l'Université de Genève, conjointement avec Genoveva Puskas, linguiste générativiste. L'objectif était de préparer les étudiants pour l'atelier Saussure-Chomsky: converging and diverging qui se tiendra en janvier 2017 dans le cadre du colloque clg2016: L'Emergence célébrant à Genève les cent ans de la parution du CLG.

$5 \mathrm{Il}$ n'est pas certain que Chomsky ait lu le CLG en traduction, comme nous le verrons plus tard. A l'époque où Chomsky commence à discuter Saussure, il n'existait qu'une seule traduction en anglais, celle de W. Baskin. Harris (1983), l'auteur de la deuxième traduction anglaise du CLG, semble penser que Chomsky a lu Saussure en anglais, puisque dans sa «Translator's Introduction» à sa propre traduction, il prétend que le travail de Baskin était si mauvais que Chomsky ne pouvait que mal interpréter Saussure!

6 Dans l'édition critique de R. Engler $C L G / E$ (dans ce cas nous citons d'abord le numéro attribué à Engler de l'extrait du CLG puis éventuellement d'autres indications). Pour les notes de Constantin du troisième cours (Constantin 2005), nous utiliserons la transcription qu'en a donnée C. Mejia dans les Cahiers Ferdinand de Saussure 58 (Constantin 2005), pp. 83-289. 
Bally et Sechehaye. Nous verrons aussi comment Chomsky a choisi de retenir ce qui l'intéressait dans l'œuvre de Saussure, quitte à laisser de côté des aspects qui auraient dû l'interpeler ${ }^{7}$.

\section{Coup d'œil sur l'histoire de la linguistique}

L'incipit du CLG et celui des notes prises par Constantin (2005) lors du troisième cours sont emblématiques de la différence entre un texte rédigé pour passer à la postérité et les premières phrases d'un enseignant annonçant le sujet de son cours. A la formulation assez définitive de Bally et Sechehaye on peut opposer à la fois par le contenu et le choix des mots l'entrée en matière de Saussure. Qu'on en juge:

Ce cours traitera de la linguistique proprement dite, et non la langue et le langage.

[...] Cette science a passé par des phases défectueuses.

Après est venue une linguistique proprement dite, consciente de son objet. (Constantin 2005: 83)

La science qui s'est constituée autour des faits de langue.... a passé par trois phases successives avant de reconnaître quel est son véritable et unique objet. (CLG: 13)

La présentation orale du cours vise à définir la linguistique, cette science nouvelle ou qui, du moins, n'avait pas encore développé une véritable conscience de son objet. Dans le CLG par contre, la science est déjà constituée et son «véritable et unique objet» est la langue. Cela contraste avec cette première phrase notée par Constantin dans laquelle Saussure, qui n'a pas encore discuté de la matière ni de l'objet de la linguistique, exclut non seulement le langage mais aussi la langue. Saussure avait déjà affirmé que de partir de la notion intuitive de langue ou de langage était potentiellement trompeur, comme en atteste la première phrase de la première page des notes prises par Riedlinger au deuxième cours de linguistique générale (19081909), et que Sechehaye avait recopiée en 1914:

La ling. n'est pas toute simple dans son principe, $<$ dans sa méthode $>$, dans l'ensemble de ses recherches, parce que la langue ne l'est pas. Au 1. abord c'est le contraire qui paraît. La langue $<$ [le langage ?] $>$ nous paraît tt près de notre main; peut-être est-elle trop près» (d'après Sofia 2015: XCVI).

Dans la publication les éditeurs n'ont donc pas jugé utile de reprendre cette cautèle.

Bally et Sechehaye spécifient parfois les choses là où Saussure se montre plus général. Ainsi, dans la revue des 'phases défectueuses' de l'histoire de la linguistique, là où Saussure regrette chez les grammairiens l'absence d'une «vue supérieure sur ce qu'est le phénomène de la langue dans son ensemble» (Constantin 2005: 83), le CLG leur reproche d'être «éloigné[s] de la pure observation», donnant à penser que cette 'vue supérieure' équivalait à de l'observation. Certaines reformulations, anodines au premier abord, peuvent même se révéler carrément problématiques. Alors que Saussure parle des «conditions où la langue vit» (Constantin 2005: 84 ) -dont les comparatistes ne se préoccupèrent pas-, les éditeurs écrivent «les conditions de la vie des langues» (CLG 18), ce qui semble entrer en contradiction avec la note - tirée du deuxième cours- figurant à la fin de ce premier chapitre de l'Introduction, dans laquelle on lit à propos des "métaphores illogiques» dont se servaient les comparatistes: "Dès lors on n'ose plus [...] parler de la 'vie des langues', etc., puisque la langue n'est pas une entité, et n'existe que dans les sujets». (CLG: 19). Il est vrai que Saussure ajoutait «il y a un certain nombre d'images qu'il faut nécessairement employer, le tout est de s'entendre» (CLG/E: 100 II R 162) que les éditeurs complètent parant ainsi à tout reproche ultérieur:

Exiger qu'on ne se serve que de termes répondant aux réalités du langage, c'est prétendre que ces réalités n'ont plus de mystères pour nous. Or, il s'en faut de beaucoup; aussi n'hésiterons-nous pas à employer à l'occasion telle des expressions qui ont été blâmées à l'époque (CLG: 19, n. 1).

7 Joseph (2002: 133-155) montre les fluctuations de Chomsky par rapport à Saussure. Cela va de the Saussurean à The Anti-Saussurean pour finir avec the Neo-Saussurean! 
Pour asseoir davantage la pensée de ce qu'ils présentent les éditeurs recourent donc parfois aux autres cours professés par Saussure voire à ses notes personnelles. Si, dans ses cahiers $\mathrm{du}$ troisième cours Constantin écrit que la grammaire comparée ne voit pas encore «l'esprit de la linguistique», le CLG restitue cela au moyen d'un passage tiré du deuxième cours: «elle ne s'est jamais préoccupée de dégager la nature de son objet d'étude. Or, sans cette opération élémentaire une science est incapable de se faire une méthode» (CLG: 16/CLG/E: 57-58). Or, c'est bien à cette entreprise que se livre Saussure dans ce troisième cours, mais seulement brièvement au début, avant de la poursuivre une fois traité le chapitre sur 'les langues' (diversité géographique, écriture et familles de langues). Un agencement que les éditeurs n'ont pas du tout retenu, comme on le sait ${ }^{8}$. Pour renforcer les propos tenus lors du troisième cours et dans l'objectif de renforcer ce qui est dit de l'objet de la linguistique, Bally et Sechehaye utilisent les notes de Saussure lui-même qu'ils reformulent quelque peu:

Bien loin que l'objet précède le point de vue, on dirait que c'est le point de vue qui précède l'objet, et d'ailleurs rien ne me dit d'avance que l'une de ces manières de considérer le fait en question soit antérieure ou supérieure aux autres.

(CLG: 23) p. 4)

Résumé plus général: voici le sens le plus général de ce que nous avons cherché à établir: Il nous est interdit en linguistique <quoique nous ne cessions de la faire> de parler ' $d$ 'une chose' à différents points de vue, <ou d'une chose en géné-ral $>$, parce que c'est le point de vue qui FAIT la chose.

(CLG/E: n. 9.2 [3295a]

Ce «point de vue qui crée l'objet» est devenu une des formules choc pour caractériser l'apport de Saussure à la linguistique ${ }^{9}$. Saussure utilise l'expression 'du point de vue de..' par exemple pour parler du point de vue panchronique (CLG: 134), et dans son cours, autre exemple, il parle par de «La diversité géographique de la langue envisagée du point de vue de ses causes» que les éditeurs gardent dans un premier temps, mais finissent par publier sous «Causes de la diversité géographique» (CLG: 270), pour des questions de style probablement, mais perdant du même coup l'idée qu'on examine le phénomène «d'un certain point de vue»! ${ }^{10}$

\section{Le circuit de la parole}

Ce nouvel exemple va concerner l'interprétation faite par les éditeurs de deux schémas qui se trouvent dans le CLG dans le deuxième alinéa: «Place de la langue dans les faits de langage» du chapitre II (intitulé «L'objet de la linguistique») de l'Introduction, alors que dans le troisième cours (Constantin 2005) ainsi que dans la Collation (Sofia 2015: 511), il se trouve dans la deuxième partie du cours, une fois le première partie sur Les langues conclue. Autre différence qui s'offre d'emblée au regard mais que nous ne pouvons reproduire ici: dans les notes de Constantin les deux dessins sont côte à côte alors que dans le CLG ils sont séparés par du texte.

Nous reprenons l'entrée en matière du CLG qui est sensiblement la même que le texte noté par Constantin (2005) mais dans une rédaction plus définitive.

Pour trouver dans l'ensemble du langage la sphère qui correspond à la langue, il faut se placer devant l'acte individuel qui permet de reconstituer le circuit de la parole. Cet acte suppose au moins deux individus; c'est

8 Comme cela a été remarqué par Godel dans les Sources manuscrites: «Le plan a été établi sur la base du troisième cours, mais l'ordre des divisions générales, indiqué et motivé par Saussure [les langues, la langue, la faculté et exercice du langage chez les individus] n’a pas été maintenu» (Godel 1957: 98).

9 V. par ex. l'Encyclopédie Larousse en ligne, l'Encyclopédie Universalis en ligne ou wikiquotes ; dans les trois, c'est la première citation de Saussure qui est donnée: http://www.larousse.fr/encyclopedie/divers/ linguistique/66057;http://www.universalis.fr/encyclopedie/langue-et-parole-linguistique https://fr.wikiquote. org/wiki/Ferdinand_de_Saussure consultées le 21 octobre 2016.

10 Toutefois, un petit pointage montre que dans le CLG le terme 'point de vue' est utilisé plus de de cinquante fois! 
le minimum exigible pour que le circuit soit complet. Soient donc deux personnes, $A$ et $B$, qui s'entretiennent:

Les deux individus sont représentés ainsi chez les étudiants et dans le CLG

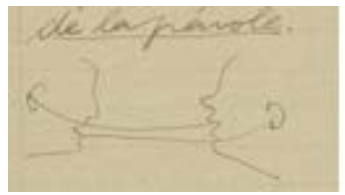

Constantin 2005: 215

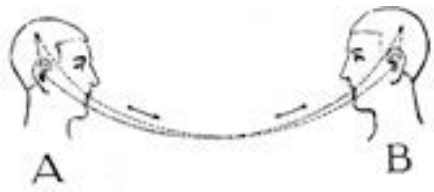

$C L G: 27$

Dans un premier temps, la Collation reprend le dessin des étudiants. Mais en voulant confier à l'imprimeur un schéma plus élaboré, les éditeurs ont voulu, semble-t-il, anticiper la schématisation que Saussure donnera dans un deuxième temps. Ce fai-sant le schéma tel qu'il apparaît dans le livre perd le lien si évident allant de la 'bouche de l'un à l'oreille de l'autre' - sans qu' on puisse savoir qui commence, donnant ainsi une image que l'on pourrait qualifier de 'mouvement perpétuel'. Dans le dessin imprimé par contre, ceci est peu lisible: le passage de la bouche de l'un à l'oreille de l'autre ne ressort pas, le trait semblant passer par le hasard de la courbe à la hauteur de la bouche. En outre, Bally et Sechehaye ont introduit un passage par le cerveau pour montrer le lieu de l'association 'image acoustique-concept' (ou vice-versa 'concept-image acoustique'), mais ce qui frappe c'est que si le lien 'oreille-cerveau' apparaît clairement, celui qui va du cerveau à la bouche ne l'est pas.

Venons-en maintenant à l'autre schéma, celui qui explicite les différentes phases du circuit; pour mémoire, dans les notes des étudiants les deux dessins étaient l'un à côté de l'autre. Ce n'est pas la seule différence.
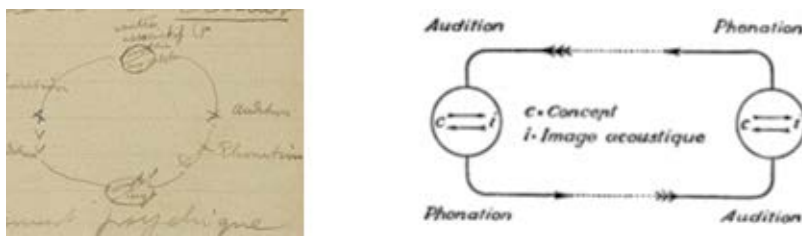

Constantin 2005: 215
Mis à part le fait que le cercle s'est transformé en une sorte de rectangle, rendant moins évidente une cyclicité11, ce qui frappe se situe au niveau de la bulle contenant 'concept/image acoustique'. Le schéma imprimé, en ne séparant pas par un trait le concept de l'image acoustique rend mieux que le dessin l'idée que l'un appelle l'autre et qu'ils sont indissociables. Par contre, contrairement à ce que Sechehaye avait transcrit dans sa Collation (Sofia: 407), le schéma imprimé ne comprend pas la mention de «centre d'associatif». D'une certaine manière, le fait que concept et image acoustique ne soient pas séparés montre bien leur union, et donc implicitement leur association. Sur le schéma, l'absence de la mention en toutes lettres du lieu de la partie psychique -le centre associatif- le démarque par rapport aux phénomènes physiologiques que sont l'audition et la phonation, mais ne donne pas tout de suite à voir qu'il est le contrepoids de ces dernières.

Une meilleure lecture de la distinction languel parole aurait pu permettre à Chomsky d'éviter de grossièrement mal interpréter ce circuit de la parole:

In a work that inaugurated the modern era of language study Ferdinand de Saussure (1916) drew a fundamental distinction between what he called langue and parole. The first is the grammatical and semantic system repre-sented in the brain of the speaker; the second is the actual acoustic output from his vocal organs and input to his ears. (Chomsky 1963: 327).

En effet, dans l'acte de parole, Saussure distingue deux parties: la partie psychique (l'utilisation du code de la langue) et le mécanisme psycho-physique qui en découle:

La parole est au contraire un acte individuel de volonté et d'intelligence, dans lequel il convient de distinguer: 1o les combinaisons par lesquelles le sujet parlant utilise le code de la langue en vue d'exprimer sa pensée personnelle; 2o le mécanisme psycho-physique qui lui permet d'extérioriser ces combinaisons. (CLG: 30-31)

11 Les étudiants du séminaire Saussure-Chomsky ont vivement plébiscité le dessin de Constantin au détriment du schéma imprimé! 
Notons que dans les notes d'étudiants comme G. Dégallier, F. Joseph ou Mme A. Sechehaye, on trouve la chose dans un ordre inversé:

1o Usage des facultés en général en vue du langage (phonation, etc.).

2o Aussi: Usage individuel du code de langue selon la pensée individuelle. (CLG/E: extr. 247, D.)

L'intervention des éditeurs permet une meilleure lecture respectant une meilleure suite des événements.

\section{Le jeu d'échecs}

Venons-en maintenant à notre dernier exemple. Il s'agit de la similarité entre le jeu d'échecs et la langue dont Chomsky retient l'idée d'une langue -la langue saussurienne, I-language pour ce qui le concerne- vue comme un ensemble de règles.

It should be noted that familiar characterizations of 'language' as a code or a game point correctly toward I-language, not the artificial construct E-language. A code is not a set of representations but rather a specific system of rules that assign coded representations to message-representations. Two codes may be different, although extensively identical in their message-code pairing that they provide. Similarly, a game is not a set of moves but rather the rule system that underlies them. The Saussurean concept of langue, although far too narrow in conception, might be interpreted as appropriate in this respect. (Chomsky 1986: 31)

Dans le CLG on trouve deux passages où cette comparaison est développée. Le premier intervient dans le chapitre III -«La linguistique statique et la linguistique évolutive»- de la première partie consacrée au Principes généraux. Le thème en est la différence et l'indépendance entre ces deux ordres de faits linguistiques. Après avoir utilisé une métaphore botanique, la coupe transversale ou, au contraire, longitudinale d'un végétal, Saussure se lance dans une autre comparaison.

Mais de toutes les comparaisons qu'on pourrait imaginer, la plus démons-trative est celle qu' on établirait entre le jeu de la langue et une partie d'échecs. De part et d'autre, on est en présence d'un système de valeurs et on assiste à leurs modifications. Une partie d'échecs est comme une réalisation artificielle de ce que la langue nous présente sous une forme naturelle.

Il développe la chose sur le plan synchronique, celui qui intéresse Chomsky:

D'abord un état du jeu correspond bien à un état de la langue. La valeur respective des pièces dépend de leur position sur l'échiquier, de même que dans la langue chaque terme a sa valeur par son opposition avec tous les autres termes.

Mais c'est la permanence des règles que le linguiste américain relève, quand bien même Saussure est en train de pointer par-là vers la diachronie-.

En second lieu, le système n'est jamais que momentané; il varie d'une posi-tion à l'autre. Il est vrai que les valeurs dépendent aussi et surtout d'une convention immuable, la règle du jeu, qui existe avant le début de la partie et persiste après chaque coup. Cette règle admise une fois pour toutes existe aussi en matière de langue; ce sont les principes constants de la sémiologie. (CLG 125-126)

Chomsky cependant n'a pas vu deux conséquences de ce rapprochement. La première concerne la valeur que chaque pièce acquiert $\mathrm{du}$ fait qu'elle relève d'un système. C'est la compréhension de ce qu'est ce dernier qui lui fait dire que le concept de langue est «far too narrow»:

Modern linguistics is much under the influence of Saussure's conception of langue as an inventory of elements (Saussure, 1916, 154, and elsewhere, fre-quently) and his preoccupation with systems of elements rather than the system of rules which were the focus of attention in traditional grammar [...]. (Chomsky 1964: 23)

12 Chomsky a peut-être bien lu le CLG en français puisque c'est à l'édition française que renvoie l'indication de page. 
Mais si chaque pièce acquiert sa valeur par opposition aux autres, il est aussi vrai que: «la valeur respective des pièces dépend de leur position sur l'échiquier», comme on peut le lire dans la citation ci-dessus. Chomsky aurait peut-être dû s'intéresser à l'idée qu'une valeur n'est pas intrinsèque mais doit toujours être vue dans un état mettant en œuvre le système de la langue. Le deuxième élément essentiel dans la théorie saussurienne est l'inclusion de cette règle (valeur des termes dans le système et les uns par rapport aux autres dans un état donné) dans les principes généraux de la sémiologie. Il ne peutêtre pas étonnant que cet aspect ait été pas-sé sous silence, parce qu'il implique l'historicité des systèmes sémiologiques, dont la langue, alors que pour Chomsky le I-language est une connaissance qui repose sur la faculté de langage -dont la Grammaire universelle (UG) est la théorieet a donc un substrat biologique.

L'autre mention du jeu d'échec est le passage auquel Chomsky fait référence dans la citation ci-dessus et qui se situe un peu plus loin dans le CLG, dans le chapitre III -«Identités, réalités valeurs»- de la deuxième partie: Linguistique synchronique. Il est d'autant plus étonnant que Chomsky n'a pas relevé l'idée de valeur que ce chapitre y est consacré.

Prenons un cavalier: est-il à lui seul un élément du jeu? Assurément non, puisque dans sa matérialité pure, hors de sa case et des autres conditions du jeu, il ne représente rien pour le joueur et ne devient élément réel et concret qu'une fois revêtu de sa valeur et faisant corps avec elle. Supposons qu'au cours d'une partie cette pièce vienne à être détruite ou égarée: peut-on la remplacer par une autre équivalente? Certainement: non seulement un autre cavalier, mais même une figure dépourvue de toute ressemblance avec celleci sera déclarée identique, pourvu qu'on lui attribue la même valeur. On voit donc que dans les systèmes sémiologiques, comme la langue, où les éléments se tiennent réciproquement en équilibre selon des règles déterminées, la notion d'identité se confond avec celle de valeur et récipro-quement. (CLG: 153).

Chomsky n’a donc pas relevé le concept de valeur mais seulement celui de règles. Là non plus il n'a pas attaché d'importance à la mention de systèmes sémiologiques et à sa conséquence: «en définitive la notion de valeur recouvre celles d'unité, d'entité concrète et de réalité» (CLG: 154). Saussure nous fait ici une démonstration qui aurait toutefois dû l'intéresser: les entités linguistiques ont une réalité qui, bien que psychique, ne s'explique qu'en fonction d'un système de valeurs qui est lui de nature historicosociale.

Il faut cependant envisager que, comme le souligne Newmeyer (2013), la lecture de Saussure par Chomsky ne visait pas tant à comprendre cet auteur que de l'utiliser à ses propres fins.

Chomsky in his 1986 book Knowledge of language appears for the first time to attribute to Saussure the idea that langue is a system of internalized rules. However, that was not Chomsky's intention. The apparent attribution was nothing more that the use of a rhetorical device of a type that Chomsky had availed himself several times in prior publications" (Newmeyer 2013: 249).

Dans ce cas, on ne saurait reprocher à Chomsky de ne pas s'être livré «Un bon exercice de lecture-profond, sérieux, systématique- [qui] peut nous amener à comprendre l'essence de la pensée d'un auteur» comme me l'écrivait Miguel Angel Mahecha Bermudez, le responsable de ce numéro (communication personnelle)!

\section{Conclusion}

Que la pensée de Saussure en matière de linguistiquegénéraleaitdonnélieuà-demultiples interprétations depuis son premier établissement jusqu'aux derniers rebondissements apportés par la mise à disposition de la Collation Sechehaye est un aspect de celle-ci qu'il convient de partager avec des étudiants. En effet, pour un auteur comme Saussure, on ne peut s'en tenir à ce qui est immédiatement donné, comme aimerait nous le faire croire la dernière publication du CLG par la maison Payot, qui non seulement a changé la pagination par rapport à l'édition mais a aussi choisi de ne pas reproduire tout l'appareil critique de De Mauro. Il convient de montrer à un public non encore initié, mais aussi d'exploré 
avec ceux qui sont déjà familiers de l'œuvre, c'est tout d'abord à quel point les interventions des éditeurs ont pu, pour le meilleur ou pour le pire, apporter de petites touches qui sont autant de modifications à la pensée de Saussure. C'est que nous avons essayé de montrer avec les deux premiers passages du CLG. Nous avons également eu à cœur de donner à voir que les références très nombreuses que Chomsky fait à des auteurs qui l'ont précédé et qu'il juge utile de convoquer -soit pour montrer en quoi il s'en rapproche ou alors s'en distingue- sont à prendre avec beaucoup de précaution: le but est de servir sa pensée plutôt que de livrer les résultats d'une lecture "profonde, sérieuse et systé-matique». Les étudiants du séminaire Reading Saussure and Chomsky ont l'occasion de mieux connaître chacun de ces deux auteurs pour mieux comprendre en quoi ils ont des intérêts communs et aussi en quoi ils divergent irrémédiablement. L'atelier Saussure-Chomsky: converging and diverging devrait apporter quelques éléments de réponses à leurs questions.

\section{Références}

Chomsky, N. (1963), "Formal Properties of Grammars", in Luce, Bush \& Gallanter (dir.), Handbook of Mathematical Psychology, London-New-York: Wiley, pp. 322-418.

Chomsky, N. (1986), Knowledge of language, NewYork : Praeger.

Constantin, E. (2005), «Linguistique générale, cours de M. le professeur de Saussure, 1910-1911», Cahiers Ferdinand de Saussure 58, pp. 83-289.

Forel, C. (2012) «Quelques aspects des traductions anglaises du CLG», Cahiers Ferdinand de Saussure 65, pp. 39-50.
Godel, R. (1957), Les sources manuscrites du Cours de linguistique générale de F. de Saussure, Genève: Droz.

Harris, R. (1983) «Translator's Introduction», in F.de Saussure (1983), Course in General Linguistics. Translated and annotated by Roy Harris, London: Duckworth.

Joseph, E. J. (2002), “Bloomfield's and Chomsky's Readings of the Cours de linguistique générale" in From Whitney to Chomsky: Essays in the history of American linguistics, Amsterdam/ Philadelphia: J. Benjamins.

NewmeyerF.J.(2013), “SomeremarksonChomsky's reading of Saussure", in Anderson, Moeschler \& F. Reboul, L'interface langage-cognition, The language-cognition interface, Genève: Droz.

Robert, Th. (2012), «Le facteur temps dans la vie de la langue: trois interprétations graphiques de la pensée saussurienne», Cahiers Ferdinand de Saussure 65, pp. 125-132.

Saussure, F.de (1968), Cours de linguistique générale, éd. critique par R. Engler, Wiesbaden: Harrassowitz ; ici : CLG/E.

Saussure, F. de (1983), Course in General Linguistics. Translated and annotated by Roy Harris, London: Duckworth.

Saussure, F.de (1972), Cours de linguistique générale, éd. critique par T. de Mauro, Paris: Payot; ici CLG.

Saussure, F. de (1959), Course in General Linguistics. Translated by W. Baskin, New York: Philosophical LibraryLibrary.

Sofia, E. (2015) La «Collation Sechehaye» du 'Cours de linguistique générale' de Ferdinand de Saussure, Louvain/Paris: Peeters. 